\title{
Osteocyte-specific WNT1 regulates osteoblast function during bone homeostasis
}

\author{
Kyu Sang Joeng, ${ }^{1}$ Yi-Chien Lee, ${ }^{1}$ Joohyun Lim, ${ }^{1}$ Yuqing Chen, ${ }^{1}$ Ming-Ming Jiang, ${ }^{1}$ Elda Munivez, ${ }^{1}$ \\ Catherine Ambrose, ${ }^{2}$ and Brendan H. Lee ${ }^{1}$
}

'Department of Molecular and Human Cenetics, Baylor College of Medicine, Houston, Texas, USA. ²Department of Orthopedic Surgery, University of Texas Health Science Center at Houston, Houston, Texas, USA.

\begin{abstract}
Mutations in WNT1 cause osteogenesis imperfecta (OI) and early-onset osteoporosis, identifying it as a key Wnt ligand in human bone homeostasis. However, how and where WNT1 acts in bone are unclear. To address this mechanism, we generated late-osteoblast-specific and osteocyte-specific WNT1 loss- and gain-of-function mouse models. Deletion of Wnt1 in osteocytes resulted in low bone mass with spontaneous fractures similar to that observed in OI patients. Conversely, Wnt1 overexpression from osteocytes stimulated bone formation by increasing osteoblast number and activity, which was due in part to activation of mTORC1 signaling. While antiresorptive therapy is the mainstay of OI treatment, it has limited efficacy in WNT1-related OI. In this study, anti-sclerostin antibody (Scl-Ab) treatment effectively improved bone mass and dramatically decreased fracture rate in swaying mice, a model of global Wnt1 loss. Collectively, our data suggest that WNT1-related OI and osteoporosis are caused in part by decreased mTORC1-dependent osteoblast function resulting from loss of WNT1 signaling in osteocytes. As such, this work identifies an anabolic function of osteocytes as a source of Wnt in bone development and homoeostasis, complementing their known function as targets of Wnt signaling in regulating osteoclastogenesis. Finally, this study suggests that Scl-Ab is an effective genotype-specific treatment option for WNT1-related OI and osteoporosis.
\end{abstract}

\section{Introduction}

Wnt signaling is a well-established pathway that regulates skeletal development and homeostasis (1). Genetic studies of LRP5 in human and mouse strongly suggest that canonical Wnt signaling regulates postnatal bone formation (2-5). Further genetic studies with $\beta$-catenin and other Wnt ligands using various mouse models provided additional evidence that corroborate the critical function of Wnt signaling in skeletal development and bone homeostasis (6-17). Several recent studies also reported that Wnt signaling directly regulates osteoclast function (18-22). Despite the established function of Wnt signaling in bone, the role of specific Wnt ligands in human bone homeostasis was not clear. Our group and others reported that heterozygous loss-of-function mutations in WNT1 can cause dominantly inherited early-onset osteoporosis, while biallelic mutations resulting in complete loss of function lead to recessively inherited osteogenesis imperfecta (OI) (23-29). The semidominant inheritance of this mutation spectrum underscores the strict temporal, spatial, and dosage requirement of this essential Wnt ligand in human bone homeostasis. Moreover, we have established a swaying mouse model (Wnt $1^{\text {sw/sw }}$ mice, which carry a mutation in Wnt1) as a murine model of WNT1-related OI (30). These human and mouse genetic studies strongly suggest that WNT1 is a major Wnt ligand regulating human bone homeo-

Related Commentary: p. 2539

Authorship note: K.S. Joeng and Y.C. Lee contributed equally to this work. Conflict of interest: The authors have declared that no conflict of interest exists. Submitted: January 3, 2017; Accepted: April 27, 2017

Reference information: J Clin Invest. 2017;127(7):2678-2688.

https://doi.org/10.1172/JCI92617. stasis; however, the mechanistic basis of WNT1 action in bone homeostasis and its cellular source and targets in bone are unclear.

Osteocytes are one of the major cell types in bone. They are differentiated from osteoblasts and embedded in bone matrix. It was originally believed that osteocytes are quiescent cells in bone matrix, but numerous studies now show that osteocytes play essential roles in bone homeostasis by regulating osteoblasts and osteoclasts (31). For example, osteocytes can regulate osteoclastogenesis by expressing RANKL and its decoy receptor osteoprotegerin (7, 32-36). Osteocytes can also regulate osteoblast differentiation by secreting sclerostin (SOST), an inhibitor of Wnt signaling (37-39). However, whether osteocytes can directly regulate bone formation as a "Wnt-sending" cell type is not clear.

Mammalian target of rapamycin complex 1 (mTORC1) signaling regulates cell metabolism, growth, proliferation, and survival $(40,41)$. mTORC1 signaling can be activated by various nutritional and environmental signals, including growth factors and amino acids. The mTORC1 complex is composed of multiple components, including mTOR (a serine/threonine kinase), RAPTOR (regulatory-associated protein of mTOR), mLST8 (an mTORassociated mammalian homolog of LST8), PRAS40 (proline-rich AKT substrate $40 \mathrm{kDa}$ ), and DEPTOR (DEP domain-containing mTOR-interacting protein). The tuberous sclerosis heterodimer (TSC1/2) negatively regulates mTORC1 signaling by inhibiting Rheb (the small GTPase) that is necessary for mTORC1 kinase activity. Rapamycin is a bacterial macrolide that inhibits mTORC1 signaling by forming a complex with FKBP12. Eukaryotic initiation factor 4E-binding protein 1 (4E-BP1) and p70 ribosomal S6 kinase 1 (S6K1) are well-known downstream targets of mTORC1 signaling. Interestingly, recent studies have shown that specific Wnt ligands may regulate osteoblast differentiation, partly through mTORC1 

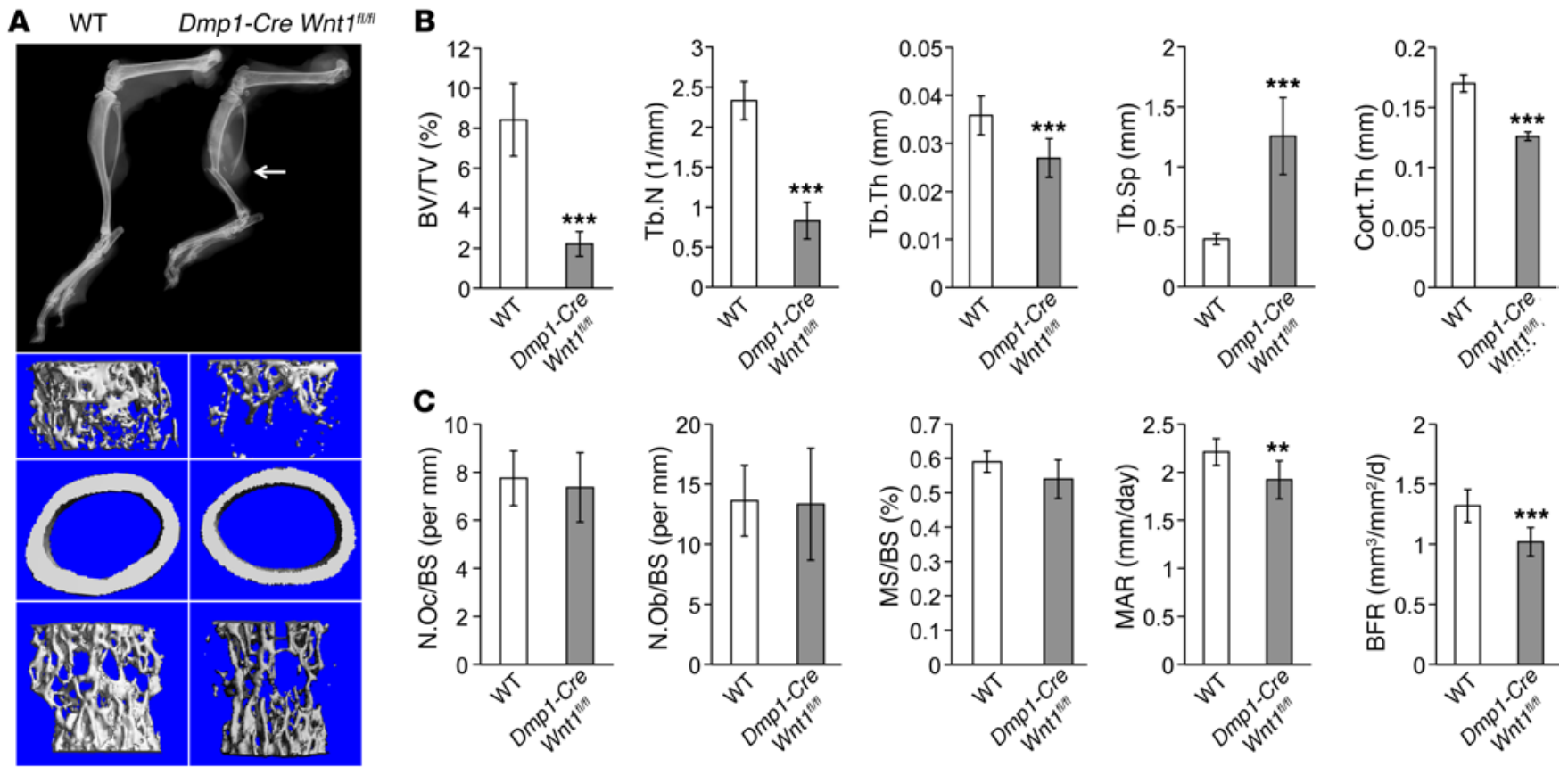

Figure 1. The phenotypes of bone-specific Wnt1 loss-of-function (Dmp1-Cre Wnt fl/fl) $^{\text {If }}$ mouse models. (A) X-ray radiograph and micro-CT ( $\mu$ CT) images of female WT and Dmp1-Cre Wnt $1^{f / f l}$ mice at 2 months old. The white arrow indicates a fracture site in the mutant mouse. (B) Quantification results of $\mu \mathrm{CT}$ analysis; femoral trabecular bone for bone volume/total volume (BV/TV), trabecular number (Tb.N), trabecular thickness (Tb.Th), and trabecular space (Tb.Sp), and cortical bone for cortical thickness (Cort.Th). Results are shown as means \pm SD ( $n=8$ per group). (C) Histomorphometric analysis of L4 vertebrae; osteoclast numbers per bone surface (N.Oc/BS), osteoblast numbers per bone surface (N.Ob/BS), mineral surface per bone surface (MS/BS), mineral apposition rate (MAR), and bone formation rate (BFR) in WT and Dmp1-Cre Wnt fl/fl mice. Results are shown as means \pm SD ( $n=6$ for WT, $n=8$ for Dmp1Cre Wnt $\left.7^{f / f}\right)$. The comparison between WT and Wnt1 loss-of-function mice is determined by Student's $t$ test. ${ }^{* *} P<0.01,{ }^{* * *} P<0.001$.

signaling $(12,42-44)$. However, whether mTORC1 is necessary for WNT1-induced osteoblast differentiation remains unclear.

Current pharmacological treatment options for OI predominantly rely on antiresorptive therapy with bisphosphonates, which inhibits bone resorption by blocking osteoclast differentiation and activity $(45,46)$. Interestingly, bisphosphonate therapy has been reported to be less effective in patients with WNT1-related OI compared with responses observed in patients with other forms of OI (47). As such, there is an unmet need of new therapeutic approaches for the treatment of WNT1-related OI and osteoporosis. Sclerostin inhibits WNT signaling by binding to and interfering with WNT ligand engagement with LDL receptor-related protein 5/6 (LRP5/6) (37-39), and has been validated as a potent therapeutic target for increasing bone formation in vivo (48-51). In fact, anti-sclerostin antibody ( $\mathrm{Scl}-\mathrm{Ab})$ is currently in phase III clinical trials as an anabolic therapy for postmenopausal osteoporosis (NCT02016716, ClinicalTrials.gov). But the efficacy of Scl-Ab for treating WNT1-related OI patients who may have significantly reduced endogenous WNT signaling is unknown. At the same time, Scl-Ab may improve bone mass and fracture rates if there is the potential of additional WNT ligands that can work redundantly in bone. Therefore, it is necessary to examine the efficacy of $\mathrm{Scl}-\mathrm{Ab}$ for treating WNT1-related OI patients as a potential, genotype-specific therapeutic approach.

In this study, we determined the bone-specific function of $W n t 1$ by generating late-osteoblast/osteocyte-specific Wnt1 lossand gain-of-function mouse models. Our pharmacological and genetic rescue experiments showed that the function of WNT1 in osteoblasts is partly mediated by mTORC1 signaling. In addition, Scl-Ab treatment improved low bone mass and dramatically decreased fracture rate in a WNT1-related OI mouse model (swaying mice, hereafter referred to as $\left.W n t 1^{s w / s w}\right)$. Overall, our data support the model wherein osteocytes serve as a source of WNT1 and the bone fragility of WNT1-related OI patients is caused in part by decreased osteoblast function due to the loss of WNT1 signaling from osteocytes to osteoblasts. Finally, this study suggests that $\mathrm{Scl}-\mathrm{Ab}$ may be an effective genotype-specific treatment option for WNT1-related patients with OI and osteoporosis.

\section{Results}

Specific deletion of Wnt1 in late osteoblasts and osteocytes caused spontaneous fractures and severe loss of bone. To elucidate the bone-specific function of $W n t 1$, we generated a Wnt 1 conditional knockout mouse model (Wnt1 $1^{f / f l}$ ) by using embryonic stem cells derived from the European Conditional Mouse Mutagenesis Program (EUCOMM) allele (Wnt1 $1^{\text {EUCOMM }}$; Supplemental Figure 1A; supplemental material available online with this article; https:// doi.org/10.1172/JCI92617DS1). We have previously shown that subsets of osteocytes are a potential source of WNT1 in bone via lineage tracing experiments (25); therefore, we deleted Wnt1 in late osteoblasts and osteocytes by generating Wnt $1^{f / / f l} \mathrm{Dmp1}$-Cre mice. Strikingly, Wnt1/f/fl Dmp1-Cre mice showed spontaneous fractures (fracture rate of 67\%) and low bone mass in both males and females, without an effect on growth or behavior (Figure 1, A and B, and Supplemental Figure 2, A and B). X-ray images of the hindlimbs showed severe osteopenia in Wnt $1^{f / f l} \mathrm{Dmp1}$-Cre mice, as 

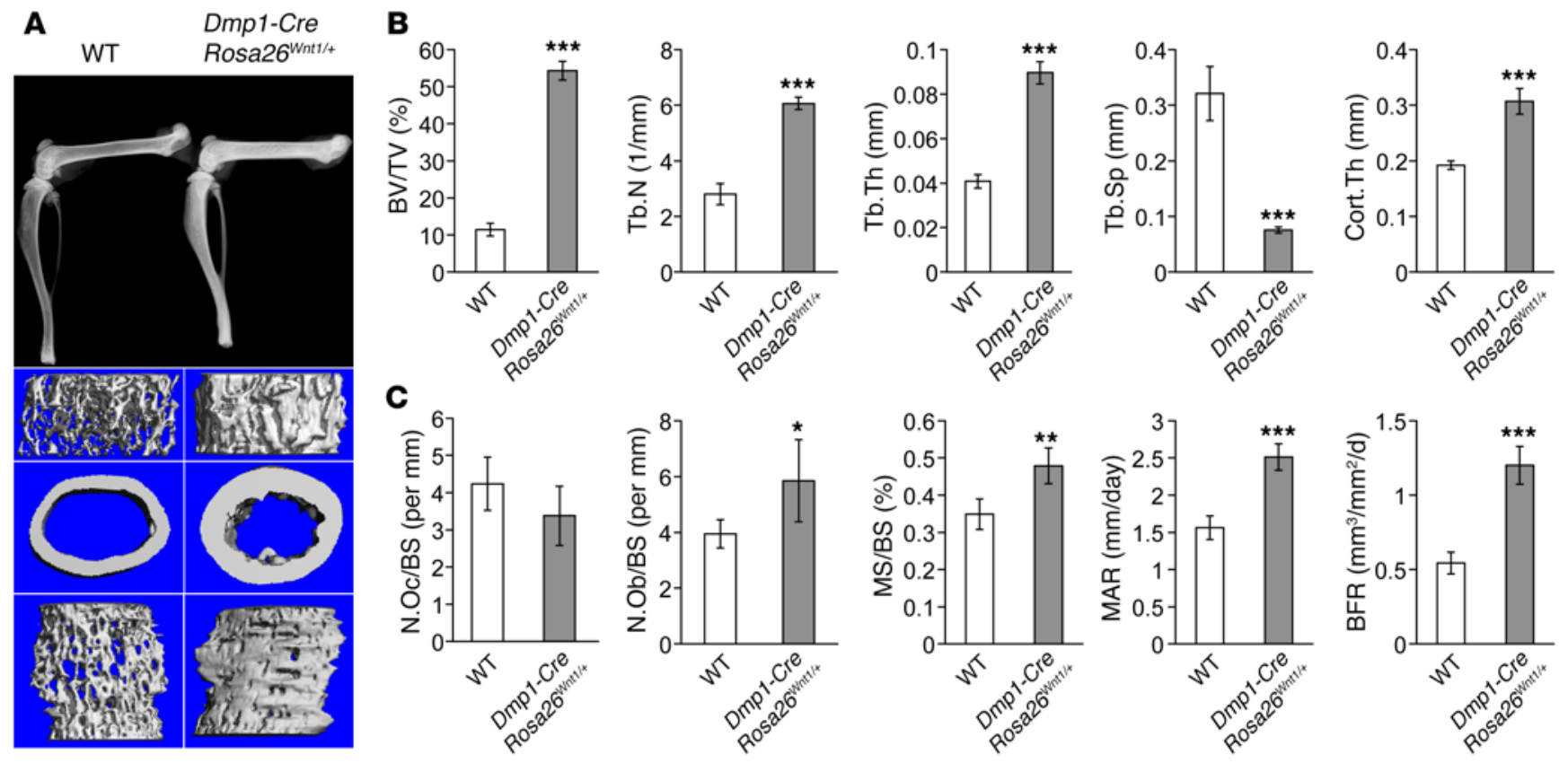

Figure 2. The phenotypes of bone-specific Wnt1 gain-of-function (Dmp1-Cre Rosa26 ${ }^{\text {Wnt1/+}}$ ) mouse models. (A) X-ray radiograph and $\mu C T$ images of female WT and Dmp1-Cre Rosa26 Wnt1/+ mice at 2 months old. (B) Quantification results of $\mu \mathrm{CT}$ analysis; femoral trabecular bone for bone volume/total volume (BV/TV), trabecular number (Tb.N), trabecular thickness (Tb.Th), and trabecular space (Tb.Sp), and cortical bone for cortical thickness (Cort.Th). Results are shown as means \pm SD ( $n=6$ per group). (C) Histomorphometric analysis of L4 vertebrae; osteoclast numbers per bone surface (N.Oc/BS), osteoblast numbers per bone surface (N.Ob/BS), mineral surface per bone surface (MS/BS), mineral apposition rate (MAR), and bone formation rate (BFR) in WT and Dmp1-Cre Rosa26 Wnt1/+ mice. Results are shown as means \pm SD ( $n=6$ per group). The comparison between WT and Wnt1 gain-of-function mice is determined by Student's $t$ test. ${ }^{*} P<0.05,{ }^{* *} P<0.01,{ }^{* * *} P<0.001$.

reflected by the increased lucency of the trabecular and cortical bones. Consistent with this observation, micro-computed tomography $(\mu \mathrm{CT})$ analysis of femurs showed a 4 -fold reduction in trabecular bone volume (BV/TV) with decreased trabecular number (Tb.N) and thickness (Tb.Th) in Wnt $1^{\text {fl/l }}$ Dmp1-Cre mice (Figure 1B). In addition, cortical bone thickness (Cort.Th) in Wnt $1^{\text {ft/fl }}$ Dmp1-Cre mice was 30\% less than that in WT mice (Figure 1B). Similarly, mutant mice displayed low bone mass in the vertebrae (Supplemental Figure 2C). To understand the cellular mechanisms of spontaneous fractures and low bone mass in Wnt $1^{1 / / /}$ Dmp1-Cre mice, we performed bone histomorphometric analysis. Interestingly, Wnt $1^{f / / l}$ Dmpl-Cre mice showed a significant decrease in mineral apposition rate and bone formation rate, but osteoblast and osteoclast numbers per bone surface were unaltered (Figure 1C). Serum CTX level was also unchanged in Wnt $1^{1 / / 7}$ Dmp1-Cre mice (Supplemental Table 1). These data indicate that Wnt1 deletion causes osteopenia and fractures primarily due to defects in osteoblast activity. Altogether, the specific deletion of Wnt 1 in late osteoblasts and osteocytes caused spontaneous fractures and severe loss of bone, which demonstrates a phenotypic overlap with the swaying mouse model, a global loss-of-function mouse model of WNT1-related OI (30). Together, these data support an essential requirement of WNT1 function in osteocytes as the dominant contributor to the pathogenesis of WNT1-related OI and osteoporosis. Moreover, current data further support that the skeletal abnormalities in WNT1-related OI patients could be caused by abnormalities in osteoblast function and that osteocytes are the main source of WNT1.
Overexpressing Wht1 in late osteoblasts and osteocytes showed a high bone mass phenotype. To gain further insight into the therapeutic potential of Wnt1 in bone mass accretion, we generated Dmp1Cre Rosa $26^{\text {Wnt1/+ }}$ mice overexpressing Wnt1 in late osteoblasts and osteocytes (Supplemental Figure 1B). In agreement with the lossof-function study, both male and female Dmp1-Cre Rosa26 Wnt1/+ mice showed a dramatic high bone mass phenotype in femurs and vertebrae (Figure 2, A-C, and Supplemental Figure 2, D-F). X-ray images of the hindlimbs showed increased bone mass in Dmp1Cre Rosa $26^{\text {Wnt1/+ }}$ mice, and $\mu \mathrm{CT}$ analysis revealed an approximately 5 -fold increase in trabecular bone mass in mutant mice, which was associated with increased trabecular number and thickness (Figure 2, A and B). Additionally, cortical bone thickness increased about $60 \%$ in mutant mice compared with WT controls (Figure 2B). Histomorphometric analysis showed significant increases in osteoblast number, mineralizing surface, mineral apposition rate, and bone forming rate, whereas osteoclast number per bone surface was unaffected in mutant mice (Figure 2C). Interestingly, serum CTX level is increased in Dmp1-Cre Rosa26 $6^{\text {Wnt1/+ }}$ mice (Supplemental Table 1), consistent with higher total osteoclast number resulting from increased total bone surface (data not shown). These data strongly suggest that the high bone mass is caused by increased osteoblast number and activity in Dmp1-Cre Rosa26 $6^{\text {Wnt } 1 /+}$ mice in opposite fashion to the conditional loss-of-function model.

Overexpression of Wnt1 increased osteoblast differentiation and mineralization in vitro. To verify the function of Wnt 1 in osteoblast differentiation and bone mineralization, we performed in vitro functional studies using a mouse stromal cell line (ST2) transduced with 
A

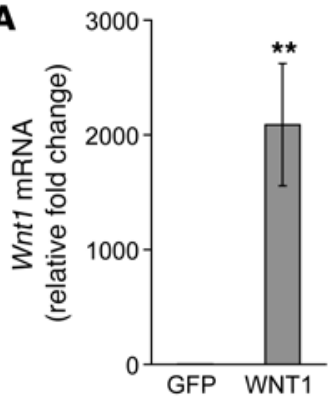

B

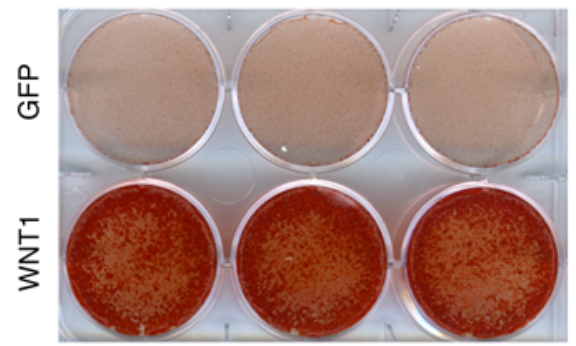

D $\longleftarrow$

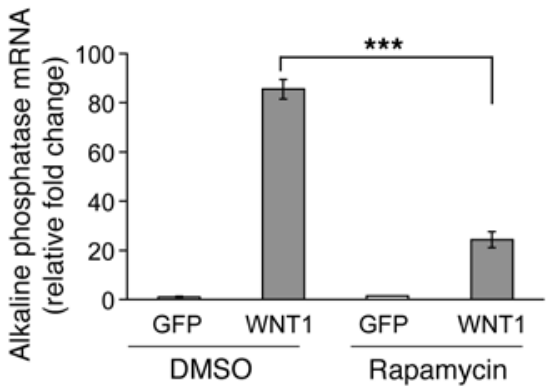

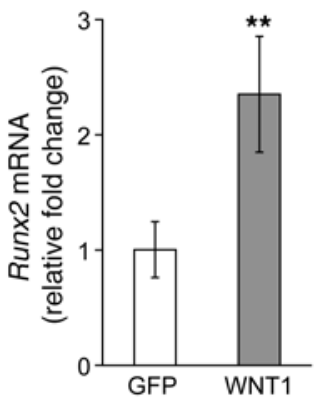

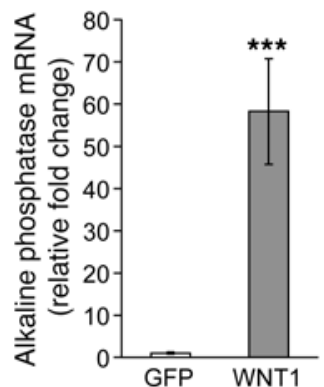

C
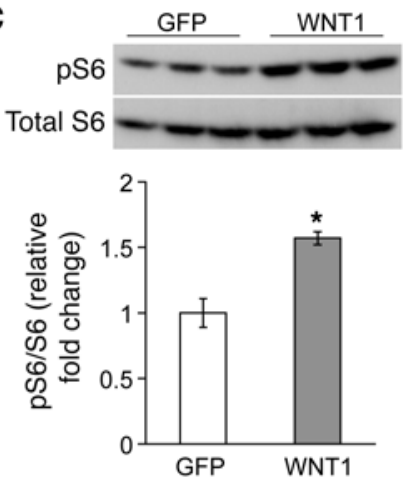

E

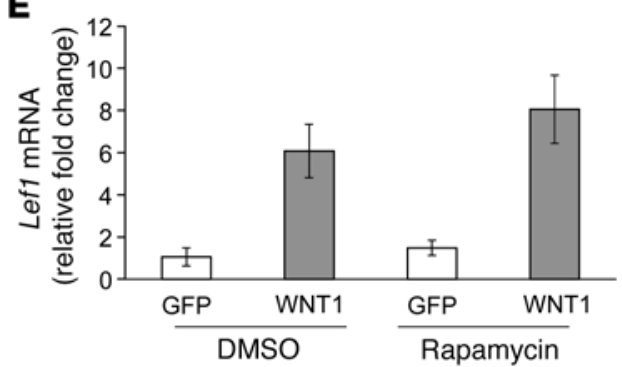

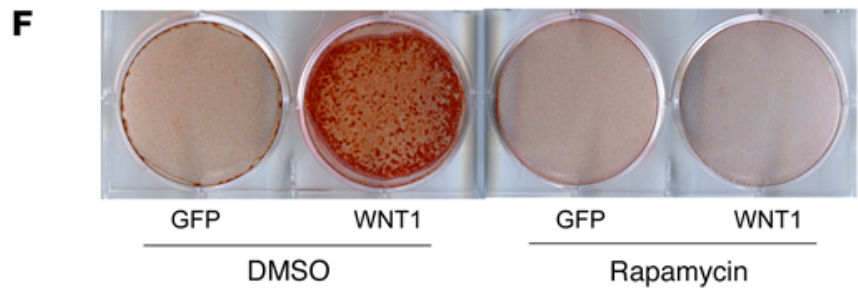

Figure 3. mTORC1 signaling mediated enhanced osteoblast differentiation and mineralization by Wnt1 overexpression in vitro. (A) Quantitative reverse transcriptase PCR (RT-PCR) of Wnt1, Runx2, alkaline phosphatase, and osteocalcin in control helper-dependent adenovirus-treated (HDAd-GFP) and WNT1 helper-dependent adenovirus-treated (HDAd-mWnt1) ST2 cells. Results are shown as fold change of the mean of control group \pm SD ( $n=3$ per group). (B) Mineralization assay by alizarin red staining on the seventh day after control virus-treated and HDAd-mWnt1-treated ST2 cells. (C) The representative Western blot analysis showed activated pS6 relative to total S6 and pAkt (Ser473) relative to total Akt of control virus- and HDAd-mWnt1-treated ST2 cells. Results are shown as fold change of the mean of control group \pm SD ( $n=3$ per group). The Western blot represents 3 individual experiments. ( $\mathbf{D}$ and E) Quantitative RT-PCR of alkaline phosphatase (D) and Lef1 (E) after control treatment (DMSO) or pharmacological inhibition of mTOR signaling by rapamycin in control virus- and HDAd-mWnt1-treated ST2 cells. Results are shown as fold change of the mean of control group \pm SD ( $n=3$ per group). (F) Mineralization assay after control treatment (DMSO) or pharmacological inhibition of mTOR signaling by rapamycin in control virus- and HDAd-mWnt1-treated ST2 cells. The comparison between control and HDAd-mWnt1-treated groups is determined by Student's $t$ test. ${ }^{*} P<0.05,{ }^{*} P<0.01,{ }^{*}{ }^{*} P<0.001$.

a helper-dependent adenoviral vector (HDAd) coding mouse Wnt1 (HDAd-mWnt1). We confirmed that the viral system efficiently overexpressed Wnt1 in ST2 cells (Figure 3A). Notably, overexpression of Wnt1 increased the expression of osteoblast differentiation markers such as Runx2, alkaline phosphatase, and osteocalcin (Figure 3A). These results suggest that osteoblast differentiation is accelerated by $W n t 1$ expression in this early and continuous exposure model. Con- sistent with enhanced osteoblast differentiation, Wnt1 overexpression markedly enhanced in vitro mineralization (Figure 3B). Based on our mouse genetics and in vitro studies, we conclude that lateosteoblast/osteocyte-derived WNT1 contributes to bone homeostasis by regulating osteoblast function. In addition, these results confirm that impaired osteoblast function due to loss of Wnt1 in osteocytes is a possible pathogenic mechanism of WNT1-related OI. 
A

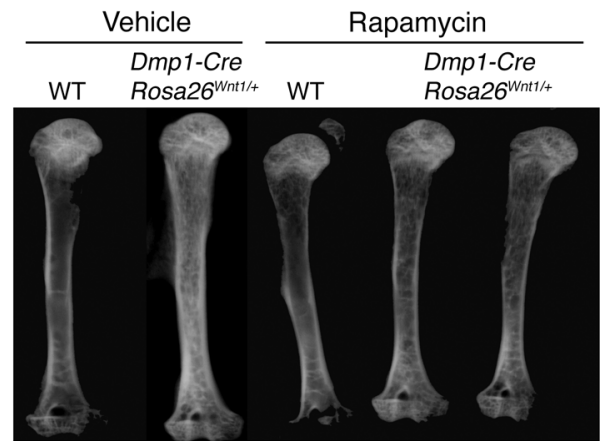

B

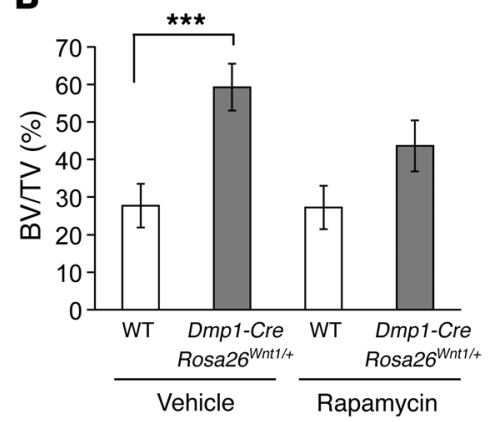

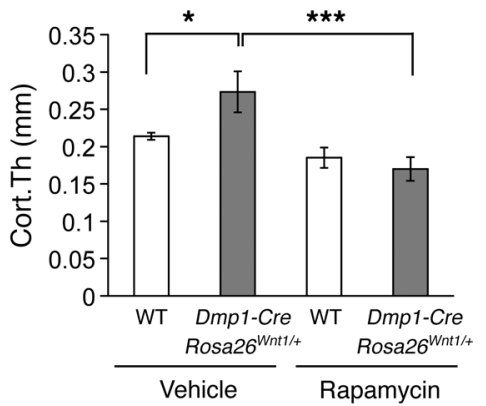
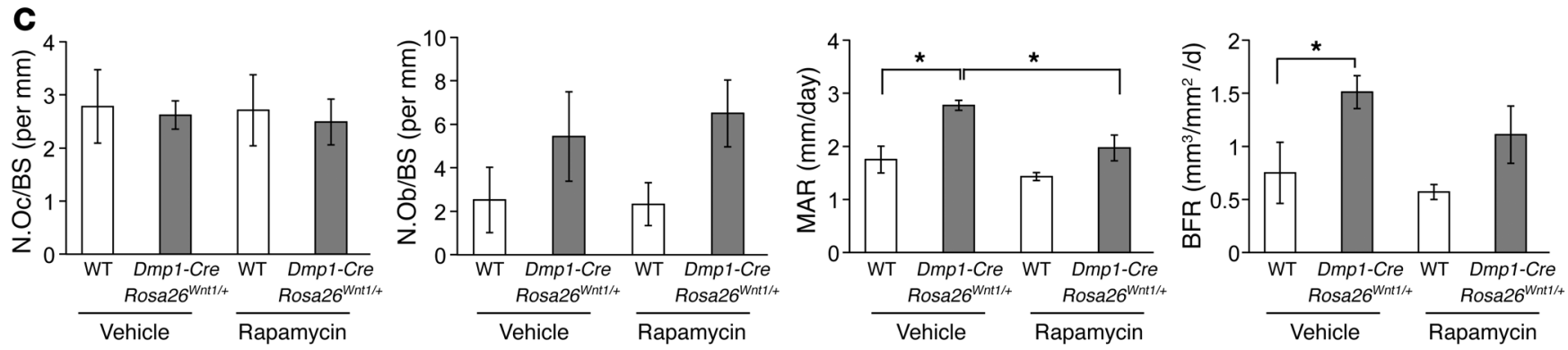

Figure 4. WNT1 gain-of-function mouse models showed significantly reduced high bone mass after treatment with the mTORC1 signaling inhibitor rapamycin. (A) X-ray radiograph of femurs of female WT and Dmp1-Cre Rosa26 $6^{\text {Wnt } /+}$ mice after vehicle control or rapamycin injections in 2-month-old mice. (B) $\mu \mathrm{CT}$ analysis of femoral trabecular bone for bone volume/total volume (BV/TV) and of cortical bone for cortical thickness (Cort.Th) in WT and Dmp1-Cre Rosa26 Wnt1/+ mice treated with vehicle control or rapamycin. Results are shown as means \pm SD ( $n=5$ for WT, $n=4$ for Dmp1-Cre Rosa26 $\left.6^{W n t 1 /+}\right)$. (C) Histomorphometric analysis of L4 vertebrae for osteoclast numbers per bone surface (N.Oc/BS), osteoblast numbers per bone surface (N.Ob/BS), mineral surface per bone surface (MS/BS), mineral apposition rate (MAR), and bone formation rate (BFR) in WT and Dmp1-Cre Rosa26 Wnt1/+ mice treated with vehicle control or rapamycin. Results are shown as means \pm SD ( $n=5$ for WT, $n=4$ for Dmp1-Cre Rosa26 $\left.{ }^{W n t 1 /+}\right)$. The comparisons between vehicle-treated WT and vehicle-treated WNT1 gain-of-function groups and between vehicle-treated and rapamycin-treated WNT1 gain-of-function groups are determined by Mann-Whitney $U$ test. ${ }^{*} P<0.05,{ }^{* * *} P<0.001$.

Phenotypes caused by gain of WNT1 signaling can be reversed by rapamycin. Previous studies by others have shown that Wnt signaling can regulate canonical $\beta$-catenin pathways as well as noncanonical targets like the mTOR pathway $(12,42-44)$. Interestingly, recent studies have demonstrated that WNT ligands may activate mTOR signaling in osteoblasts to affect their differentiation $(12,42)$. Consistent with these studies, we found that Wnt1 overexpression significantly increased phosphorylation of S6, a major downstream target of mTORC1, but not phosphorylation of Akt, a major downstream target of mTORC2 (Figure 3C). Based on these results, we hypothesized that mTORC1 signaling may mediate WNT1 function in osteoblast differentiation and mineralization. To test this hypothesis, we performed a pharmacological rescue experiment by treating ST2 cells overexpressing Wnt 1 with rapamycin, an inhibitor of mTORC1 signaling (52). Rapamycin treatment significantly reduced the expression of Wnt1-induced alkaline phosphatase (Figure 3D). We also examined the expression of Axin2 and Lef1, major downstream targets of $\beta$-catenindependent Wnt signaling. The expression level of Axin 2 was not detectable (data not shown), while the expression of Lef1 was significantly induced by Wnt1 expression (Figure 3E). However, the Wnt1-induced Lef1 expression was not rescued by rapamycin treatment (Figure 3E). Consistent with rescued expression of alkaline phosphatase, rapamycin treatment significantly reduced Wnt1induced in vitro mineralization (Figure $3 \mathrm{~F}$ ). Collectively, these data suggest that mTORC1 mediates Wnt1-induced osteoblast differentiation and in vitro mineralization in a $\beta$-catenin-independent manner. To verify these results in vivo, we performed a pharmacological rescue experiment by treating Dmp1-Cre Rosa $26^{\text {Wntl } /+}$ mice with rapamycin. Interestingly, 1 month of rapamycin treatment significantly reversed the high bone mass phenotype of Dmp1-Cre Rosa $26^{\text {Wnt1/+ }}$ mice (Figure 4, A and B). X-ray radiography revealed a reduction in trabecular bone and cortical bone thickness of rapamycin-treated Dmp1-Cre Rosa26 $6^{\text {Wnt } 1++}$ mice (Figure $4 \mathrm{~A}) . \mu \mathrm{CT}$ parameters showed that rapamycin fully normalized cortical bone thickness and partially reduced trabecular bone volume (Figure 4B), suggesting a differential impact of mTORC1 signaling on WNT1 function in trabecular versus cortical bone compartments. Histomorphometric analysis showed that inhibition of MTORC1 in the WNT1 gain-of-function model was associated with reduced mineral apposition rate and bone formation rate, but not changes in osteoclast and osteoblast number (Figure 4C). This pharmacological rescue experiment supports that mTORC1 partially mediates the gain of WNT1 signaling in osteoblasts.

Genetic activation of $m$ TORC1 signaling rescued the low bone mass phenotype of the swaying mice. To test the physiological relevance of Wnt1-induced mTORC1 signaling, we determined whether genetic activation of mTORC1 signaling could rescue the low bone mass caused by loss of Wnt1 signaling in bone. To this end, we activated MTORC1 signaling in late osteoblasts/osteocytes by deleting $T s c 1$, a negative regulator of $\mathrm{mTORC} 1$, in $W n t 1^{\mathrm{sw} / \mathrm{sw}}$ mice $\left(T s c 1^{A / / l} W_{n t} 1^{s w / s w}\right.$ Dmp1-Cre). As expected, Tsc $1^{f / / l}$ Wnt $1^{s w / s w}$ Dmp1- 

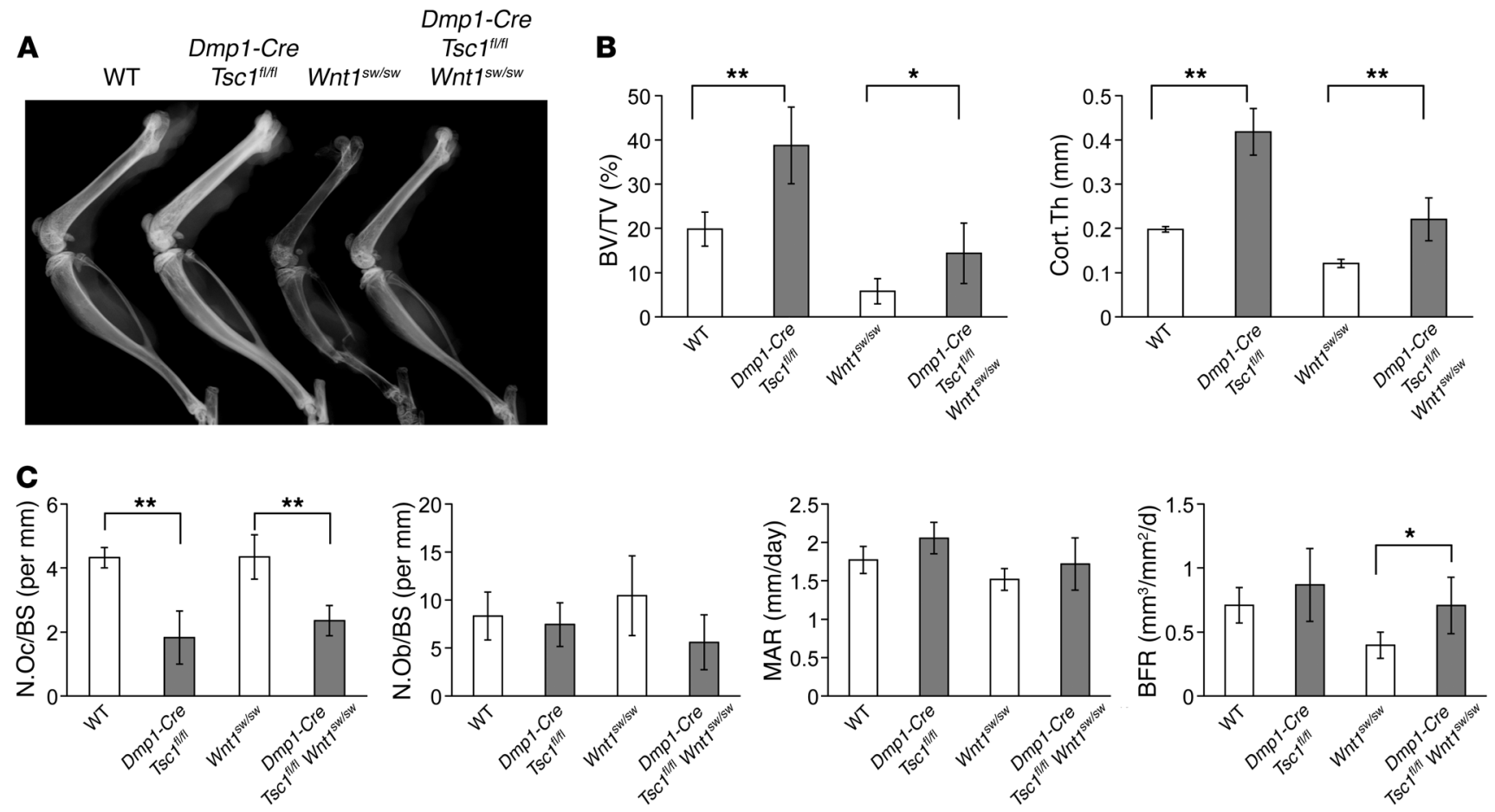

Figure 5. Genetic activation of mTORC1 signaling partially rescued the low bone mass phenotype of the Wnt ${ }^{\text {sw/sw }}$ models. (A) X-ray radiograph of femurs

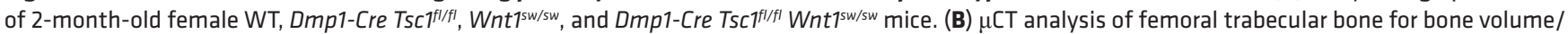

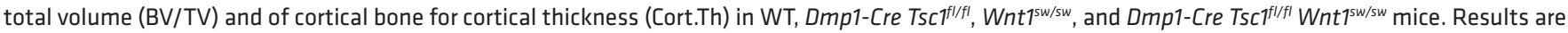
shown as means \pm SD ( $n=6$ for WT, $n=5$ for Dmp1-Cre Tsc $7^{f / f l}, n=4$ for Wnt $7^{\text {sw/sw }}, n=6$ for Dmp1-Cre Tsc $7^{f l / f l}$ Wnt $7^{\text {sw/sw }}$ mice). (C) Histomorphometric analysis of L4 vertebrae for osteoclast numbers per bone surface (N.Oc/BS), osteoblast numbers per bone surface (N.Ob/BS), mineral surface per bone surface (MS/BS), mineral apposition rate (MAR), and bone formation rate (BFR) in WT, Dmp1-Cre Tsc $1^{f l / f l}, W n t 1^{s w / s w}$, and Dmp1-Cre Tsc $7^{f l / f l}$ Wnt $7^{\text {sw/sw }}$ mice. Results are shown as means \pm SD ( $n=6$ for WT, $n=5$ for Dmp1-Cre Tsc $1^{f l / f 1}, n=6$ for Wnt ${ }^{\text {sw/sw }}, n=6$ for Dmp1-Cre Tsc $1^{f l / f l}$ Wnt $7^{\text {sw/sw }}$ mice). The comparisons between WT and Dmp1-Cre Tsc $7^{f l f l}$ and between Wnt $7^{\text {sw/sw }}$ and Dmp1-Cre Tsc $f^{f l / f l}$ Wnt $1^{\text {sw/sw }}$ are determined by Mann-Whitney $U$ test. ${ }^{*} P<0.05,{ }^{* *} P<0.01$.

Cre mice no longer suffered spontaneous fractures and had higher trabecular and cortical bone parameters when compared with $W n t 1^{15 / / s w}$ mice (Figure $5 \mathrm{~A}$ ). $\mu \mathrm{CT}$ analysis showed partial rescue of trabecular bone and full rescue of cortical bone thickness in $T s c 1^{f / / f l}$ Wnt $1^{1 \mathrm{w} / \mathrm{sw}}$ Dmp1-Cre mice (Figure 5B). Furthermore, histomorphometric analysis revealed that the rescue phenotype was associated with increased bone formation rate and also decreased osteoclast numbers (Figure 5C). Based on our mouse rescue studies and in vitro experiments, we conclude that mTORC1 partially mediates the physiological function of WNT1 signaling in regulating bone formation and mineralization.

Scl-Ab treatment significantly improved the low bone mass phenotypes of swaying mice. To test both the therapeutic potential of anti-sclerostin antibody (Scl-Ab) for WNT1-related OI patients and the potential for redundancy of Wnt ligand function in bone, we administered Scl-Ab subcutaneously at a dose of $25 \mathrm{mg} / \mathrm{kg}$ to $W n t 1^{s w / s w}$ mice twice a week from 2 to 8 weeks of age. Remarkably, Scl-Ab administration in $W n t 1^{s w / s w}$ mice caused a significant improvement in fracture rate from $90 \%$ to $12.5 \%$ (Table 1), as well as increased bone mass (Figure $6 \mathrm{~A}$ ). $\mu \mathrm{CT}$ analysis showed that $\mathrm{Scl}-\mathrm{Ab}$-treated $\mathrm{Wnt} 1^{\mathrm{sw} / \mathrm{sw}}$ mice exhibited a significant increase in trabecular bone volume and cortical thickness in both femur and lumbar spine (Figure 6B and Supplemental Figure 3). Scl-Ab treatment significantly increased osteoblast activity as indicated by the increased mineralized surface, mineral apposition rate, and bone formation rate (Figure 6C). More importantly, 3-point bending analysis confirmed that the Scl-Ab-treated $W n t 1^{s w / s w}$ mice had significantly increased bone ultimate load, stiffness, and post-yield energy compared with untreated $W n t 1^{\text {sw/sw }}$ mice (Figure 6D). Taken together, these results indicate that Scl-Ab treatment significantly improved the bone quantity and quality of $W n t 1^{\text {sw/sw }}$ mice.

\section{Discussion}

Our current studies significantly advance our understanding of the function of Wnt ligand in bone homeostasis and the pathogenic mechanism of WNT1-related skeletal disorders. Given the CNS phenotype found in WNT1-related OI patients and the Wnt $1^{\text {sw/sw }}$ mouse model $(23-29,53)$, the bone fragility of WNT1-related OI could be caused by loss of WNT1 function in bone as well as in other organs. In addition, the extremely low expression of Wnt1 in bone has also hindered determination of the tissue-specific function of WNT1. Therefore, our mouse genetic studies using a conditional model provide strong evidence that WNT1 specifically functions in bone. We generated a conditional mouse model deleting Wnt1 in late osteoblasts/osteocytes using the Dmp1-Cre transgenic line. Interestingly, Dmp1-Cre-specific deletion of Wnt1 causes spontaneous fractures and low bone mass, recapitulating the phenotypes of $W n t 1^{\text {sw/sw }}$ mice. These data strongly suggest that 
Table 1. Fracture rate of WT and Wnt $\mathbf{T}^{\text {sw/sw }}$ mice treated with vehicle control or Scl-Ab

\begin{tabular}{|c|c|c|c|}
\hline Genotype & Treatment & Number of mice & Fracture rate $(\%)$ \\
\hline WT & Vehicle & 7 & 0 \\
\hline WT & Scl-Ab & 4 & 0 \\
\hline$W n t t^{5 w / s w}$ & Vehicle & 10 & 90 \\
\hline Wnt $\overbrace{}^{\text {ww/sw }}$ & Scl-Ab & $8^{A}$ & 12.5 \\
\hline
\end{tabular}

The comparison of $\mathrm{Wnt} 1^{\mathrm{sw} / \mathrm{sw}}$ mice treated with vehicle control versus $\mathrm{Scl}-\mathrm{Ab}$ is determined by Fisher exact test. ${ }^{A} P<0.01$.

the bone fragility in $W n t 1^{s w / s w}$ mice and WNT1-related OI patients is mainly caused by loss of WNT1 function in bone.

Our Wnt1 loss- and gain-of-function studies suggest that osteoblasts are the major target cell of WNT1 signaling. The low bone mass phenotype in the loss-of-function study is mainly associated with decreased osteoblast activity, while the high bone mass phenotype in the gain-of-function study is associated with increased osteoblast number and activity. It is worth noting that osteoblast number was only affected in the gain-of-function study, which may be partly because of overexpression of Wnt1. Nonetheless, we conclude that late-osteoblast/osteocyte-derived Wnt1 contributes to bone homeostasis by regulating osteoblast function.

Our study also provides functional evidence that osteocytes are the major source of WNT1. Our previous lineage-tracing experiment using the transgenic Wnt1-Cre transgenic mice supports the hypothesis that osteocytes are a potential source of WNT1 in bone, although we could not rule out nonspecific effects of the transgene in those experiments (25). Our current Dmp1-Cre-specific Wnt1 loss-of-function study provides strong evidence supporting the idea that the major skeletal source of WNT1 is a subset of osteocytes in bone, as suggested by the lineage-tracing experiment. This is also consistent with the extremely low expression of Wnt1 in bone. The specificity of Dmp1-Cre to late osteoblasts and osteocytes does not rule out the possibility that the late osteoblasts may also express Wnt1. But we found only GFP-positive osteocytes and no GFP-positive osteoblasts in our previous lineage-tracing experiment (25). This strongly suggests that osteocytes are a major source of WNT1 in bone rather than osteoblasts. Further investigation of Wnt1 deletion with early-osteoblast Cre lines will further elucidate the function of WNT1 in early osteoblast lineage cells.

Our finding of osteocytes as a source of WNT1 ligand suggests that osteocytes could have a dual role as a sender and receiver of Wnt ligands during bone homeostasis (Figure 6E). As sending cells, osteocytes stimulate osteoblastogenesis by triggering Wnt signaling in osteoblasts. This is consistent with previous studies in which mice with bone-specific $L r p 5$ and Lrp6 knockout showed osteoblast-specific phenotypes $(54,55)$. Our study and those of others strongly suggest that the regulation of osteoblastogenesis by osteocytes could be mediated by a $\beta$-catenin-independent pathway like mTOR signaling $(12,42)$. As receiving cells, osteocytes can modulate osteoclastogenesis by triggering cell-autonomous $\beta$-catenin-dependent signaling. Consistent with this notion, osteocyte-specific $\beta$-catenin knockout mice show predominantly an osteoclast phenotype (33). In addition, previous studies showed that osteocytes regulate osteoclastogenesis by secreting RANKL and osteoprotegerin that are downstream targets of canonical $\beta$-catenin-dependent WNT signaling (7, 32-36). Our current study and other previous studies also strongly suggest that there is specificity between WNT ligands and downstream cell signaling targets $(10,12,14,42)$. Hence, the osteocyte can be seen increasingly as the central regulator of bone mass. As a receiver of Wnt signaling, it regulates osteoclastogenesis in a $\beta$-catenin-dependent fashion. As a sender cell of Wnt signaling, it controls anabolic aspects of osteoblast function by targeting mTORC1, though we cannot rule out additional roles in early stages of osteoblast differentiation. This last function together with its production of sclerostin shows that it can also serve as a negative and positive regulator of osteoblast differentiation and function, respectively (Figure 6E). Recent studies also showed that Wnt signaling could directly regulate osteoclastogenesis (18-22). Further studies will be required to elucidate the direct regulation of osteoclasts by osteocyte-derived Wnt signaling. The code for the underlying ligand-cell specificity likely integrates both temporal and spatial requirements that help to explain the complexity of both cellular (osteoblastic versus osteoclastic) and tissue compartment (trabecular versus cortical, and appendicular versus axial) phenotypes observed in different genetic models of Wnt signaling components. With no doubt, additional study and improved genetic reagents will be required to resolve this Wnt signaling code in the skeleton.

Scl-Ab treatment significantly improved the bone quantity and quality of $W n t 1^{s w / s w}$ mice. The rescued bone phenotypes of $W n t 1^{s w / s w}$ mice strongly suggest that other WNT ligands, including excellent candidates such as WNT16, WNT10b, and WNT7b, can compensate for loss of Wnt1 in $W n t 1^{s w / s w}$ mice (10-17). Despite the potential redundancy of multiple WNT ligands that affect bone formation, the incomplete restoration of bone volume by Scl-Ab treatment in $W n t 1^{s w / s w}$ mice suggests that WNT1 clearly has a critical function in bone homeostasis. Overall, this study supports the potential utility of Scl-Ab in the management of OI patients with WNT1 mutations. Further investigation to determine the efficacy of Scl-Ab in human WNT1-related OI patients and early-onset osteoporosis will be required for clinical application.

\section{Methods}

Animals. Embryonic stem cells carrying knockout-first Wnt1 allele $\left(W n t 1^{\text {EUCOMM }}\right.$ ) were obtained from the European Conditional Mouse Mutagenesis Program (EUCOMM) and injected into embryos in house. Wnt $1^{\text {ЕUсомM }}$ mice were crossed with Rosa26-Flippase (Flp) mice to delete $\beta$-gal and the neo cassette in order to generate the conditional knockout allele $\left(W n t 1^{f l / f)}\right.$. Rosa2 $6^{\text {Wnt1/+ }}$ mice were provided by Thomas Carroll and Andrew McMahon (56), and Dmp1-Cre transgenic mice were provided by Jian Feng (57). The swaying (Wnt $1^{15 / / s w}$ ) mouse (58, 59) and $T s c 1^{f / f l}$ mouse (60) models were obtained from the Jackson Laboratory. Rapamycin was purchased from LC Laboratories, and subcutaneous rapamycin $(4 \mathrm{mg} / \mathrm{kg})$ was administered daily to female Rosa26 $6^{\text {Wnt1/+ }}$ mice for a month. The sclerostin-neutralizing antibody (Scl-Ab) was provided by Amgen Inc. and UCB Pharma. Scl-Ab was diluted 1:10 in PBS to a concentration of $3.6 \mathrm{mg} / \mathrm{ml}$ for administration. Female $W n t 1^{s w / s w}$ and WT mice were randomly assigned and treated with subcutaneous Scl-Ab $(25 \mathrm{mg} / \mathrm{kg})$ or the control vehicle (PBS) twice per week, starting at 3 weeks old, for 6 weeks and were eutha- 
A

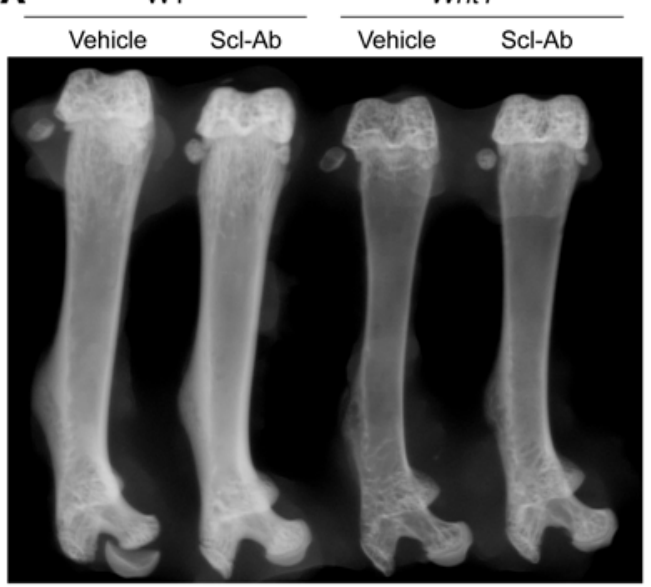

B

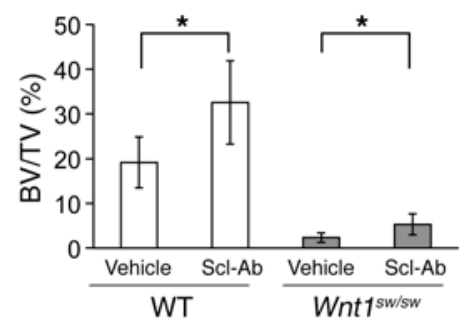

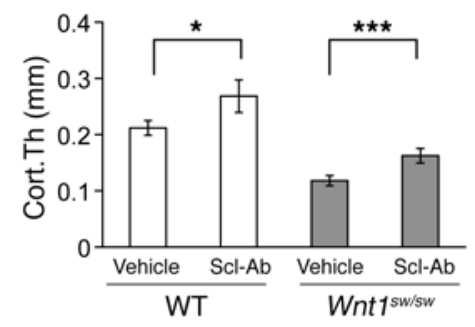
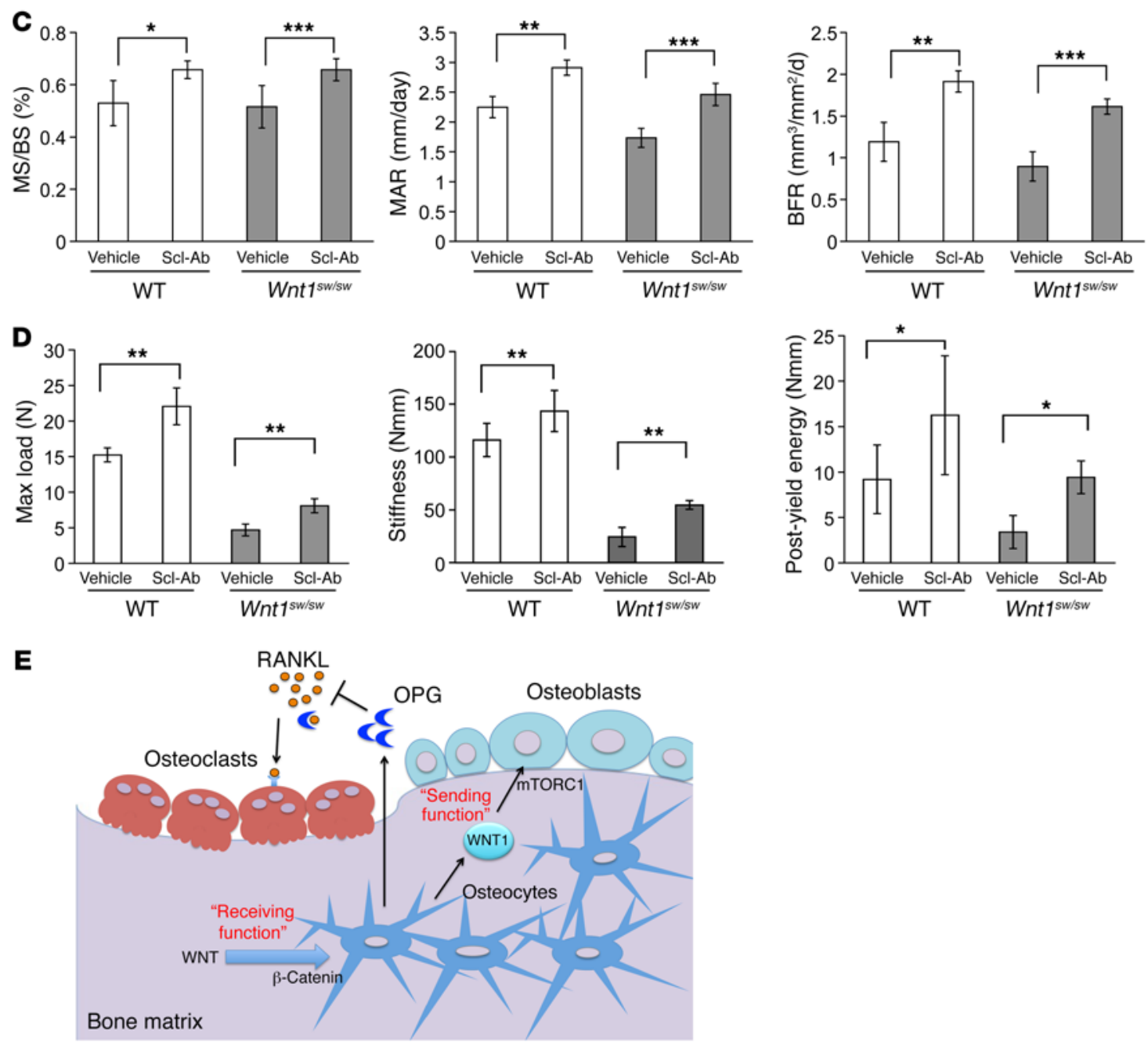

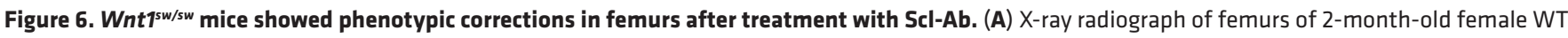
and $W_{n t} t^{\mathrm{sw} / \mathrm{sw}}$ mice treated with vehicle control or sclerostin-neutralizing antibody (Scl-Ab). (B) $\mu \mathrm{CT}$ analysis of femoral trabecular bone for bone volume/ total volume (BV/TV) and of cortical bone for cortical thickness (Cort.Th) in WT and Wnt $7^{\text {sw/sw }}$ mice treated with vehicle control or Scl-Ab. Results are shown as means \pm SD ( $n=7$ for WT, $n=3$ for WT with Scl-Ab, $n=8$ for Wnt $7^{\text {sw/sw }}, n=7$ for Wnt $7^{\text {sw/sw }}$ with Scl-Ab). (C) Histomorphometric analysis of L4 vertebrae for mineral surface per bone surface (MS/BS), mineral apposition rate (MAR), and bone formation rate (BFR) in WT and Wnt $7^{\text {sw/sw }}$ mice treated with vehicle control or Scl-Ab. Results are shown as means \pm SD ( $n=6$ for WT, $n=4$ for WT with Scl-Ab, $n=9$ for Wnt ${ }^{\text {sw/sw }}, n=7$ for Wnt $7^{\text {sw/sw }}$ with Scl-Ab). (D) Biomechanical testing results by 3-point bending assay for maximum load, stiffness, and post-yield energy of femurs in WT and Wnt ${ }^{\text {sw/sw }}$ mice treated with vehicle control or Scl-Ab. Results are shown as means \pm SD ( $n=7$ for WT, $n=4$ for WT with Scl-Ab, $n=8$ for Wnt $7^{\mathrm{sw} / \mathrm{sw}}, n=8$ for Wnt $7^{\mathrm{sw} / \mathrm{sw}}$ with Scl-Ab). The comparisons of WT mice with vehicle versus Scl-Ab treatment and Wnt $t^{\text {sw/sw }}$ mice with vehicle versus Scl-Ab treatment are determined by Mann-Whitney $U$ test. ${ }^{*} P<0.05,{ }^{* *} P<0.01,{ }^{* *} P<0.001$. (E) Schematic model of osteocyte function as a sender and receiver of Wnt signaling. OPG, osteoprotegerin. 
nized at 8 weeks old. Fracture rate was defined by callus formation in tibia during final dissection of mouse at 8 weeks old. Either unilateral or bilateral fracture in a mouse was counted as 1 incident fracture. No blinding was possible during treatment because Scl-Ab or control vehicle was injected according to group allocation. In all subsequent analyses, the investigators were blinded to genotype and treatment group. Calcein ( $250 \mu$ g i.p.) was injected first and followed by alizarin red $(800 \mu$ i.p.) injections with a 5-day interval before euthanasia to assess the parameters of dynamic bone histomorphometry. Spines and right femurs were collected and fixed in $10 \%$ formalin for 48 hours for subsequent radiography and $\mu \mathrm{CT}$ imaging. After imaging, spines were embedded in plastic for histomorphometry. Left femurs were wrapped in saline-soaked gauze and stored at $-20^{\circ} \mathrm{C}$ for biomechanical testing.

Both male and female mice were characterized for Wnt1 gain- and loss-of-function studies. Male mouse data are presented in Supplemental Figure 2. For the mTORC1 genetic rescue experiment, female mice were mainly characterized at 2 months of age. We also characterized male mice using $\mu \mathrm{CT}$ ( $n=3$, data not shown). We performed the rapamycin rescue experiments to be consistent with the mTORC1 genetic rescue experiment. Therefore, female mice were characterized at 2 months of age. For the Scl-Ab treatment experiment, female mice were used to prevent artificial fractures in the swaying mouse model, which occurred more frequently in male mice, likely due to their propensity for physical movement. The bone phenotypes for the $\mathrm{Scl}-\mathrm{Ab}$ rescue experiment were characterized in 2-month-old mice.

ST2 cell culture. The bone marrow-derived stromal cell line ST2 (61) was provided by the laboratory of Fanxin Long (Department of Orthopedic Surgery, Washington University School of Medicine, St. Louis, Missouri, USA) (the cells were not recently further profiled or tested for mycoplasma contamination). ST2 cells were cultured in growth medium ( $\alpha$-MEM HyClone with $10 \%$ FBS, $100 \mathrm{U} / \mathrm{ml}$ penicillin, and $100 \mu \mathrm{g} / \mathrm{ml}$ streptomycin). GFP and WNT1 helper-dependent adenoviruses were generated as previously described (62). The viruses were added to the medium (500 viral particles per cell) for 24 hours. After virus transfection, the medium was replaced with mineralization medium (growth medium with $500 \mu \mathrm{M}$ ascorbic acid and $10 \mathrm{mM} \beta$-glycerophosphate) (day 0). The mineralization medium was changed every 2 days. Rapamycin in DMSO (20 nM) was added to the mineralization medium and fed to the virus-infected ST2 cells from day 0. ST2 cells were collected for real-time PCR on day 4. Alizarin red staining was performed on day 10.

Quantitative real-time reverse transcriptase PCR. Total RNA from virus-transfected ST2 cells was extracted with Trizol reagent (Invitrogen). The Superscript III reverse transcriptase PCR (RT-PCR) system (Invitrogen) was used to synthesize cDNA. We performed quantitative RT-PCR on a LightCycler 96 (Roche) using gene-specific primers and SYBR Green I reagent (Roche). $\beta_{2}$-Microglobulin was used as the reference gene for normalizing cDNA concentrations.

Immunoblotting and antibodies. Protein from virus-transfected ST2 cells was extracted with Laemmli buffer containing 5\% $\beta$-mercaptoethanol. The pS6 (2211, Cell Signaling), pAkt (Ser473; 4060, Cell Signaling), S6 (2217, Cell Signaling), and Akt (4691, Cell Signaling) monoclonal antibodies were used with a 1:1,000 dilution in Tris-buffered saline and Tween 20 containing 5\% BSA. Quantification of the density of each band was performed by Image software (NIH).

Radiography, $\mu C T$ imaging, and bone histomorphometry. Radiography was performed with the Xpert 80 (Kubtec) system, and $\mu \mathrm{CT}$ was conducted with the Scanco $\mu \mathrm{CT}-40$ system (55-peak kilovoltage and $145-\mu \mathrm{A} x$-ray source). A standardized region of the distal femoral metaphysis and the L4 vertebrae were scanned at $16-\mu \mathrm{m}$ resolutions. Mouse spine samples were then embedded in plastic and sectioned with tungsten carbide blades. Trichrome staining and tartrate-resistant acid phosphatase staining were performed for visualizing osteoblasts and osteoclasts, respectively. Calcein and alizarin red double labeling was used to study dynamic histomorphometry. Results were quantified with the Bioquant Osteo 2014 Image Analysis System for evaluation of bone formation and resorption parameters.

Biomechanical testing by 3-point bending. Left femurs were tested by 3-point bending, with a span of $6 \mathrm{~mm}$, using an Instron 5848 device (Instron Inc.). All the femurs were tested wet at room temperature. They were preloaded to $1 \mathrm{~N}$ at a rate of $0.05 \mathrm{~N} / \mathrm{s}$ for 5 seconds. Following preloading, the femurs were compressed to failure at a rate of 0.1 $\mathrm{mm} / \mathrm{s}$. Load and displacement data were captured at a rate of $40 \mathrm{~Hz}$ using Bluehill Software (Instron). Maximum load was determined by finding the highest load values recorded before the specimen failed. Stiffness was determined by the steepest slope of the linear portion of the load and displacement curve. The yield point was calculated using a $0.2 \%$ offset from the steepest slope. Using the trapezoidal numerical integration method, energy to failure was calculated as the area under the load-displacement curve and was divided into the elastic energy (up to the yield point) and plastic energy (from the yield point until the failure point).

Serum CTX-1 level by ELISA. Blood samples were collected from 2-month-old mice by retro-orbital bleeding under anesthesia before euthanasia. Serum was separated by centrifuge in the tube with serum separator (BD Microtainer) and stored at $-80^{\circ} \mathrm{C}$. The bone resorption marker C-terminal telopeptide of type I collagen (CTX-1) was measured in serum. The assay was performed using a CTX-1 ELISA kit (RatLaps, Immunodiagnostic Systems) according to the manufacturer's instructions. Data were analyzed with SigmaPlot 11.0 (Systat Software Inc.).

Statistics. For comparisons between 2 groups following normal distribution, we performed 2-tailed Student's $t$ tests with a significance level of 0.05. For comparisons among 4 groups, and for those observations that did not follow normal distribution, we performed the Kruskal-Wallis test to detect heterogeneity among groups and applied the Mann-Whitney $U$ test for multiple comparisons between groups. Bonferroni adjustment was applied for multiple comparisons. Each $P$ value was compared with the critical $P$ value divided by the total number of comparisons made to determine whether differences between groups were significant. To determine the initial sample size per group of mice, we performed power analysis, which showed that a group size of 6 mice is required to detect a minimal difference of $20 \%$ in bone mass (BV/TV) by $\mu \mathrm{CT}$ between Dmp1-Cre Rosa2 $6^{\text {Wnt1/+ }}$ mice and WT mice. We used the statistical software R (3.2.4) for all statistical analyses.

Study approval. The animal care and use for this study have been approved by the Institutional Animal Care and Use Committee of Baylor College of Medicine (Houston, Texas, USA).

\section{Author contributions}

KSJ, YCL, and BHL designed the research studies. KSJ, YCL, JL, YC, MMJ, and EM conducted the experiments. KSJ and YCL analyzed the data. CA conducted the biomechanical studies. KSJ, YCL, and BHL wrote the manuscript. 


\section{Acknowledgments}

The authors thank Brian Dawson and Carrie Jiang for technical assistance. We also appreciate Thomas Carroll, Andrew McMahon, and Jian Feng for providing Rosa26-Wnt1 and Dmp1-Cre mouse lines. This work was supported by the Baylor College of Medicine (BCM) Intellectual and Developmental Disabilities Research Center (HD024064) of the Eunice Kennedy Shriver National Institute of Child Health and Human Development, the BCM Advanced Technology Cores with funding from the NIH (AI036211, CA125123, and RR024574), the Rolanette and
Berdon Lawrence Bone Disease Program of Texas, and the BCM Center for Skeletal Medicine and Biology. Research funding to authors includes NIH grants PO1 HD22657 (to BHL), PO1 HD070394 (to BHL), K01 AR069002 (to KSJ), and the Rising Star Award of American Society for Bone and Mineral Research (to KSJ).

Address correspondence to: Brendan H. Lee, Baylor College of Medicine, One Baylor Plaza, Houston, Texas 77030, USA. Phone: 713.798.8835; Email: blee@bcm.edu.
1. Baron R, Kneissel M. WNT signaling in bone homeostasis and disease: from human mutations to treatments. Nat Med. 2013;19(2):179-192.

2. Babij P, et al. High bone mass in mice expressing a mutant LRP5 gene. J Bone Miner Res. 2003;18(6):960-974.

3. Boyden LM, et al. High bone density due to a mutation in LDL-receptor-related protein 5. N Engl JMed. 2002;346(20):1513-1521.

4. Gong Y, et al. LDL receptor-related protein 5 (LRP5) affects bone accrual and eye development. Cell. 2001;107(4):513-523.

5. Little $\mathrm{RD}$, et al. A mutation in the LDL receptorrelated protein 5 gene results in the autosomal dominant high-bone-mass trait. Am J Hum Genet. 2002;70(1):11-19.

6. Day TF, Guo X, Garrett-Beal L, Yang Y. Wnt/ $\beta$-catenin signaling in mesenchymal progenitors controls osteoblast and chondrocyte differentiation during vertebrate skeletogenesis. Dev Cell. 2005;8(5):739-750.

7. Glass DA, et al. Canonical Wnt signaling in differentiated osteoblasts controls osteoclast differentiation. Dev Cell. 2005;8(5):751-764.

8. Hilton MJ, Tu X, Cook J, Hu H, Long F. Ihh controls cartilage development by antagonizing Gli3, but requires additional effectors to regulate osteoblast and vascular development. Development. 2005;132(19):4339-4351.

9. Hu H, Hilton MJ, Tu X, Yu K, Ornitz DM, Long F. Sequential roles of Hedgehog and Wnt signaling in osteoblast development. Development. 2005;132(1):49-60.

10. Bennett CN, et al. Regulation of osteoblastogenesis and bone mass by Wnt10b. Proc Natl Acad Sci US A. 2005;102(9):3324-3329.

11. Bennett $\mathrm{CN}$, et al. Wnt10b increases postnatal bone formation by enhancing osteoblast differentiation. JBone Miner Res. 2007;22(12):1924-1932.

12. Chen J, et al. WNT7B promotes bone formation in part through mTORC1. PLoS Genet. 2014;10(1):e1004145.

13. Kemp JP, et al. Phenotypic dissection of bone mineral density reveals skeletal site specificity and facilitates the identification of novel loci in the genetic regulation of bone mass attainment. PLoS Genet. 2014;10(6):e1004423.

14. Movérare-Skrtic S, et al. Osteoblast-derived WNT16 represses osteoclastogenesis and prevents cortical bone fragility fractures. Nat Med. 2014;20(11):1279-1288.

15. Niu T, et al. Identification of IDUA and WNT16 phosphorylation-related non-synonymous polymorphisms for bone mineral density in meta-analyses of genome-wide association studies. J Bone Miner Res. 2016;31(2):358-368.

16. Stevens JR, Miranda-Carboni GA, Singer MA, Brugger SM, Lyons KM, Lane TF. Wnt10b deficiency results in age-dependent loss of bone mass and progressive reduction of mesenchymal progenitor cells. J Bone Miner Res. 2010;25(10):2138-2147.

17. Todd $\mathrm{H}$, et al. Wnt16 is associated with agerelated bone loss and estrogen withdrawal in murine bone. PLoS One. 2015;10(10):e0140260.

18. Albers J, et al. Canonical Wnt signaling inhibits osteoclastogenesis independent of osteoprotegerin. JCell Biol. 2013;200(4):537-549.

19. Otero $\mathrm{K}$, et al. TREM2 and $\beta$-catenin regulate bone homeostasis by controlling the rate of osteoclastogenesis. Jimmunol. 2012;188(6):2612-2621.

20. Ruiz P, Martin-Millan M, Gonzalez-Martin MC, Almeida M, González-Macias J, Ros MA. CathepsinKCre mediated deletion of $\beta$ catenin results in dramatic loss of bone mass by targeting both osteoclasts and osteoblastic cells. Sci Rep. 2016;6:36201.

21. Wei W, et al. Biphasic and dosage-dependent regulation of osteoclastogenesis by $\beta$-catenin. $\mathrm{Mol}$ Cell Biol. 2011;31(23):4706-4719.

22. Weivoda MM, et al. Wnt signaling inhibits osteoclast differentiation by activating canonical and noncanonical cAMP/PKA pathways. J Bone Miner Res. 2016;31(1):65-75.

23. Fahiminiya S, Majewski J, Mort J, Moffatt P, Glorieux FH, Rauch F. Mutations in WNT1 are a cause of osteogenesis imperfecta. JMed Genet. 2013;50(5):345-348.

24. Keupp K, et al. Mutations in WNT1 cause different forms of bone fragility. Am J Hum Genet. 2013;92(4):565-574.

25. Laine CM, et al. WNT1 mutations in early-onset osteoporosis and osteogenesis imperfecta. $\mathrm{N} \mathrm{Engl}$ JMed. 2013;368(19):1809-1816.

26. Pyott SM, et al. WNT1 mutations in families affected by moderately severe and progressive recessive osteogenesis imperfecta. Am J Hum Genet. 2013;92(4):590-597.

27. Mäkitie RE, et al. Skeletal characteristics of WNT1 osteoporosis in children and young adults. J Bone Miner Res. 2016;31(9):1734-1742.

28. Liu Y, et al. Genotype-phenotype analysis of a rare type of osteogenesis imperfecta in four Chinese families with WNT1 mutations. Clin Chim Acta. 2016;461:172-180.

29. Faqeih E, Shaheen R, Alkuraya FS. WNT1 mutation with recessive osteogenesis imperfecta and profound neurological phenotype. J Med Genet 2013;50(7):491-492.

30. Joeng KS, et al. The swaying mouse as a model of osteogenesis imperfecta caused by WNT1 mutations. Hum Mol Genet. 2014;23(15):4035-4042.

31. Plotkin LI, Bellido T. Osteocytic signalling pathways as therapeutic targets for bone fragility. Nat Rev Endocrinol. 2016;12(10):593-605.

32. Holmen SL, et al. Essential role of beta-catenin in postnatal bone acquisition. J Biol Chem. 2005;280(22):21162-21168.

33. Kramer I, et al. Osteocyte Wnt/beta-catenin signaling is required for normal bone homeostasis. Mol Cell Biol. 2010;30(12):3071-3085.

34. Nakashima T, et al. Evidence for osteocyte regulation of bone homeostasis through RANKL expression. Nat Med. 2011;17(10):1231-1234.

35. Piemontese M, Xiong J, Fujiwara Y, Thostenson JD, O'Brien CA. Cortical bone loss caused by glucocorticoid excess requires RANKL production by osteocytes and is associated with reduced OPG expression in mice. Am J Physiol Endocrinol Metab. 2016;311(3):E587-E593.

36. Xiong J, et al. Osteocytes, not osteoblasts or lining cells, are the main source of the RANKL required for osteoclast formation in remodeling bone. PLoS One. 2015;10(9):e0138189.

37. Ellies DL, et al. Bone density ligand, Sclerostin, directly interacts with LRP5 but not LRP5G171V to modulate Wnt activity. J Bone Miner Res. 2006;21(11):1738-1749.

38. Li X, et al. Sclerostin binds to LRP5/6 and antagonizes canonical Wnt signaling. J Biol Chem . 2005;280(20):19883-19887.

39. Semënov M, Tamai K, He X. SOST is a ligand for LRP5/LRP6 and a Wnt signaling inhibitor. J Biol Chem. 2005;280(29):26770-26775.

40. Laplante M, Sabatini DM. mTOR signaling in growth control and disease. Cell. 2012;149(2):274-293.

41. Zoncu R, Efeyan A, Sabatini DM. mTOR: from growth signal integration to cancer, diabetes and ageing. Nat Rev Mol Cell Biol. 2011;12(1):21-35.

42. Esen E, Chen J, Karner CM, Okunade AL, Patterson BW, Long F. WNT-LRP5 signaling induces Warburg effect through mTORC2 activation during osteoblast differentiation. Cell Metab. 2013;17(5):745-755

43. Inoki K, et al. TSC2 integrates Wnt and energy signals via a coordinated phosphorylation by AMPK and GSK3 to regulate cell growth. Cell. 2006;126 (5):955-968.

44. Shang YC, Chong ZZ, Wang S, Maiese K. Prevention of $\beta$-amyloid degeneration of microglia 
by erythropoietin depends on Wnt1, the PI 3-K/ mTOR pathway, Bad, and Bcl-xL. Aging (Albany NY). 2012;4(3):187-201.

45. Fleisch H. Bisphosphonates: mechanisms of action. Endocr Rev. 1998;19(1):80-100.

46. Hughes DE, MacDonald BR, Russell RG, Gowen M. Inhibition of osteoclast-like cell formation by bisphosphonates in long-term cultures of human bone marrow. J Clin Invest. 1989;83(6):1930-1935.

47. Palomo T, et al. Skeletal characteristics associated with homozygous and heterozygous WNT1 mutations. Bone. 2014;67:63-70.

48. Jacobsen CM, et al. Targeting the LRP5 pathway improves bone properties in a mouse model of osteogenesis imperfecta. J Bone Miner Res. 2014;29(10):2297-2306.

49. Roschger A, et al. Effect of sclerostin antibody treatment in a mouse model of severe osteogenesis imperfecta. Bone. 2014;66:182-188.

50. Sinder BP, et al. Adult Brtl/+ mouse model of osteogenesis imperfecta demonstrates anabolic response to sclerostin antibody treatment with increased bone mass and strength. Osteoporos
Int. 2014;25(8):2097-2107.

51. Grafe I, et al. Excessive transforming growth factor- $\beta$ signaling is a common mechanism in osteogenesis imperfecta. Nat Med. 2014;20(6):670-675.

52. Ballou LM, Lin RZ. Rapamycin and mTOR kinase inhibitors. JChem Biol. 2008;1(1-4):27-36.

53. Aldinger KA, et al. Variable brain phenotype primarily affects the brainstem and cerebellum in patients with osteogenesis imperfecta caused by recessive WNT1 mutations. J Med Genet. 2016;53(6):427-430.

54. Cui Y, et al. Lrp5 functions in bone to regulate bone mass. Nat Med. 2011;17(6):684-691.

55. Riddle RC, et al. Lrp5 and Lrp6 exert overlapping functions in osteoblasts during postnatal bone acquisition. PLoS ONE. 2013;8(5):e63323.

56. Carroll TJ, Park JS, Hayashi S, Majumdar A, McMahon AP. Wnt9b plays a central role in the regulation of mesenchymal to epithelial transitions underlying organogenesis of the mammalian urogenital system. Dev Cell. 2005;9(2):283-292.

57. Lu Y, Xie Y, Zhang S, Dusevich V, Bonewald
LF, Feng JQ. DMP1-targeted Cre expression in odontoblasts and osteocytes. J Dent Res. 2007;86(4):320-325.

58. Lane PW. Mouse news lett. 1967;36:40.

59. Thomas KR, Musci TS, Neumann PE, Capecchi MR. Swaying is a mutant allele of the protooncogene Wnt-1. Cell.1991;67(5):969-976.

60. Kwiatkowski DJ, et al. A mouse model of TSC1 reveals sex-dependent lethality from liver hemangiomas, and up-regulation of p70S6 kinase activity in Tsc1 null cells. Hum Mol Genet. 2002;11(5):525-534.

61. Tong J, Kishi H, Matsuda T, Muraguchi A. A bone marrow-derived stroma cell line, ST2, can support the differentiation of fetal thymocytes from the CD4+ CD8+ double negative to the CD4+ CD8+ double positive differentiation stage in vitro. Immunology. 1999;97(4):672-678.

62. Suzuki M, Cela R, Clarke C, Bertin TK, Mouriño S, Lee B. Large-scale production of high-quality helper-dependent adenoviral vectors using adherent cells in cell factories. Hum Gene Ther. 2010;21(1):120-126. 